\title{
Isotope effects and bond softening in intense-laser-field multiphoton dissociation of $\mathbf{H}_{2}^{+}$
}

\author{
Salvador Miret-Artés \\ Instituto de Matematica y Fisica Fundamental, Consejo Superior de Investigaciones Cientificas, Serrano 123, 28006 Madrid, Spain \\ Osman Atabek \\ Laboratoire de Photophysique Moléculaire, Université Paris-Sud, Batiment 213, Centre d'Orsay, 91405 Orsay, France
}

(Received 26 July 1993)

\begin{abstract}
Isotope effects in the $\mathrm{H}_{2}{ }^{+}-\mathrm{D}_{2}{ }^{+}$fragmentation by intense laser fields offer the possibility of a multiphoton interpretation of the bond-softening mechanism. Surprisingly, the calculations indicate that the one-photon dissociation of $\mathrm{D}_{2}{ }^{+}$is favored with respect to that of $\mathrm{H}_{2}{ }^{+}$. This cannot be understood, as has previously been done, by a single-photon mechanism following tunneling through a lowered potential barrier, obviously more transparent for the lighter $\mathrm{H}_{2}{ }^{+}$. It is rather a competition between this single-photon mechanism and a five-photon mechanism which is suggested for a more realistic interpretation.

PACS number(s): 42.50.Hz, 33.80.Gj, 33.80.Wz, 34.50.Rk
\end{abstract}

An increasing amount of attention has recently been devoted to the photodissociation dynamics of molecules in intense laser fields [1]. Molecular hydrogen ion represents the model system for these studies because of its simple and well-known structure, involving within the Born-Oppenheimer approximation only two electronic states, namely the ${ }^{1} \Sigma_{g}^{+}\left(1 s \sigma_{g}\right)$ ground and the ${ }^{1} \Sigma_{u}^{+}\left(2 p \sigma_{u}\right)$ repulsive excited states. The "nonresonant" dissociation with $329.7 \mathrm{~nm}$ radiation leads, when the intensities range between $10^{10}$ and $10^{14} \mathrm{~W} / \mathrm{cm}^{2}$, to abovethreshold-dissociation (ATD), bond-softening (BS), and vibrational-trapping (VT) mechanisms. The absorption of more photons than the minimum number needed for dissociation results in a series of peaks separated by the photon kinetic energy (ATD) [2-5]. Molecular potentials distort, via the strong dipole interaction, as the laser intensity is increased, causing the field-induced avoided crossing gap to open up. This results in an unstability of any ion with vibrational energy within the gap (BS) [4-6]. In some specific cases, the laser may, on the contrary, confine the system in a finite spatial region by trapping it in a bound vibrational level (VT) $[5,7]$.

Isotopes $\left(\mathrm{H}_{2}^{+}, \mathrm{D}_{2}{ }^{+}\right)$may show quite different behaviors with respect to nonadiabatic radiative couplings, such as barrier lowerings and trappings which monitor the aforementioned multiphoton mechanisms [8]. Recently, they have experimentally been addressed by Yang et al. [9] who measured different abovethreshold dissociation branching ratios for $\mathrm{H}_{2}^{+}$and $\mathrm{D}_{2}^{+}$ over a wide range of laser intensities $\left(8 \times 10^{11}-4 \times 10^{13}\right.$ $\mathrm{W} / \mathrm{cm}^{2}$ for $532-$ and $527-\mathrm{nm}$ wavelengths and 50 -ps and 10-ns pulses). Their results are in qualitative agreement with the bond-softening model and show, in particular, a higher two- versus one-photon dissociation ratio for the heavier isotope.

In this Brief Report we analyze the dissociation of $\mathbf{H}_{2}{ }^{+}$ and its isotope $\mathrm{D}_{2}{ }^{+}$when subjected to intense continuous-wave cw lasers $\left(10^{8}-10^{14} \mathrm{~W} / \mathrm{cm}^{2}\right)$ for the widely studied $329.7-\mathrm{nm}$ wavelength corresponding to a resonant three-photon absorption. A special emphasis is put on a detailed understanding of the bond-softening mechanism with respect to the total number of photons it involves. As will be shown, the different behaviors of the isotopes may somehow enlighten the discussion concerning the competition between the two possible fragmentation pathways: either a one-photon process through a barrier-lowering mechanism or a five-photon process, which is nothing but a three-photon absorption followed by two consecutive photon emissions $[10,11]$. The initial state for all isotopes is the $(v=0, j=1)$ rovibrational level of the ground electronic potential. As has often been the case in recent works, any change in the rotation quantum number due to the interaction with the field is neglected [7]. It is worthwhile to point out that, for this level, even at $10^{14} \mathrm{~W} / \mathrm{cm}^{2}$, ionization still remains negligible [12]. Thus, molecular stability with respect to dissociation is not affected by the ionization. The potentialenergy curves are given Morse-type representations [13]. The calculations are done in the so-called radiative-field gauge (interaction in velocity form) [14] with the electronic transition moment also taken from Ref. [13]. The method which is used is a time-independent full collisional treatment based on the introduction of two artificial channels, as first suggested by Bandrauk and co-workers [15] and as recently implemented for the $\mathrm{H}_{2}{ }^{+}$photodissociation case in a close-coupled-equation formalism [14]. This treatment is a generalization of Shapiro's work [16], the first open artificial channel aiming to transform the otherwise half-collision situation into a full collision and the second closed artificial channel being the true initial unperturbed (zero-field) molecular state, describing the preparation step. The power of the method relies upon the summation over field-induced resonances (even in the case of overlapping) carried out in an indirect way, from the scattering amplitude between the artificial entrance channel and the final physical continuum [14]. 
The outcome of the calculations are the relative probabilities (or branching ratios) to reach fragmentation channels with three-, two-, or one-photon net absorptions. The results, requiring the introduction of several Floquet blocks for convergence to be achieved, as a function of the field strength ranging from $10^{8}$ to $10^{14} \mathrm{~W} / \mathrm{cm}^{2}$, are displayed in Fig. 1 together with the diabatic and adiabatic potentials of the two main blocks illustrating the different channels under consideration. As in previous calculations $[10,14]$, four regimes are depicted with respect to the intensity.

(i) For weak fields $\left(I<2 \times 10^{9} \mathrm{~W} / \mathrm{cm}^{2}\right)$, although with very low efficiency, a one-photon mechanism prevails, ending up in channel $\left|2 p \sigma_{u}, n\right\rangle$.

(ii) At intermediate intensities $\left(2 \times 10^{9}\right.$ $\mathrm{W} / \mathrm{cm}^{2}<I<3 \times 10^{12} \mathrm{~W} / \mathrm{cm}^{2}$ ) the dissociation basically proceeds through the absorption of three photons, i.e., through channel $\left|2 p \sigma_{u}, n-2\right\rangle$.

(iii) The strong-field regime $\left(3 \times 10^{12}\right.$ $\mathrm{W} / \mathrm{cm}^{2}<I<7 \times 10^{13} \mathrm{~W} / \mathrm{cm}^{2}$ ) is dominated by a fourphoton ATD process; namely the simultaneous absorption of three photons followed by the emission of one photon leading to a net number of two absorbed photons and ending up in channel $\left|1 s \sigma_{g}, n-1\right\rangle$.

(iv) At very high intensities $\left(I>7 \times 10^{13} \mathrm{~W} / \mathrm{cm}^{2}\right)$ it is again the one-photon channel which is dominating. An interesting question, which has already been addressed, is the understanding of the underlying mechanism. An early interpretation relies upon a lowering and flattening of the adiabatic potential barrier at the avoided curve crossing occurring between the field-dressed ground and excited electronic potentials $[3,4,14]$. This is mainly a singlephoton-absorption process and fragmentation results from tunneling or bond softening. But it has also been pointed out that another five-photon mechanism can compete with the previous one; namely, the initial absorption of three photons followed by the emission of two photons ending up in a net one-photon dissociation channel $[10,11]$. We claim that this discussion may at least partly be clarified by referring to an isotope effect.

The analysis of the different behaviors of the isotopes is carried out at five intensities pertaining to the intermediate-, strong-, and very strong-field regimes as indicated by the arrows in Fig. 1. Table I gathers the results in terms of the branching ratios $P_{1}, P_{2}$, and $P_{2}$ towards dissociation channels corresponding to the net absorption of one, two, or three photons, respectively. A measure of the efficiency of the dissociation via threephoton (or two-photon) versus the two-photon (or onephoton) channels is the ratio $R_{32}$ (or $R_{21}$ ), i.e., $R_{i j}=P_{i} / P_{j}$. Their values are indicated in the last column of Table I. As an interpretative tool, we are referring to the simple Landau-Zener (LZ) semiclassical theory by recalling that the LZ probability $W_{i j}^{\mathrm{LZ}}$ for a transition between diabatic channels $i$ and $j$ is merely given by [17]

$$
W_{i j}^{\mathrm{LZ}}=1-\exp \left[-\frac{2 \pi V_{i j}^{2}}{h v\left|\Delta F_{i j}\right|}\right],
$$

where $V_{i j}, \Delta F_{i j}$, and $v$ are the coupling (radiative in the present case), the difference of slopes of the potentials, and the classical velocity at the crossing point. Although not valid for high intensities and in particular in cases where the coupling is not localized at the curve crossing region, this formula indicates, at least, that the heavier isotope $\mathrm{D}_{2}{ }^{+}$with less velocity behaves more adiabatical-

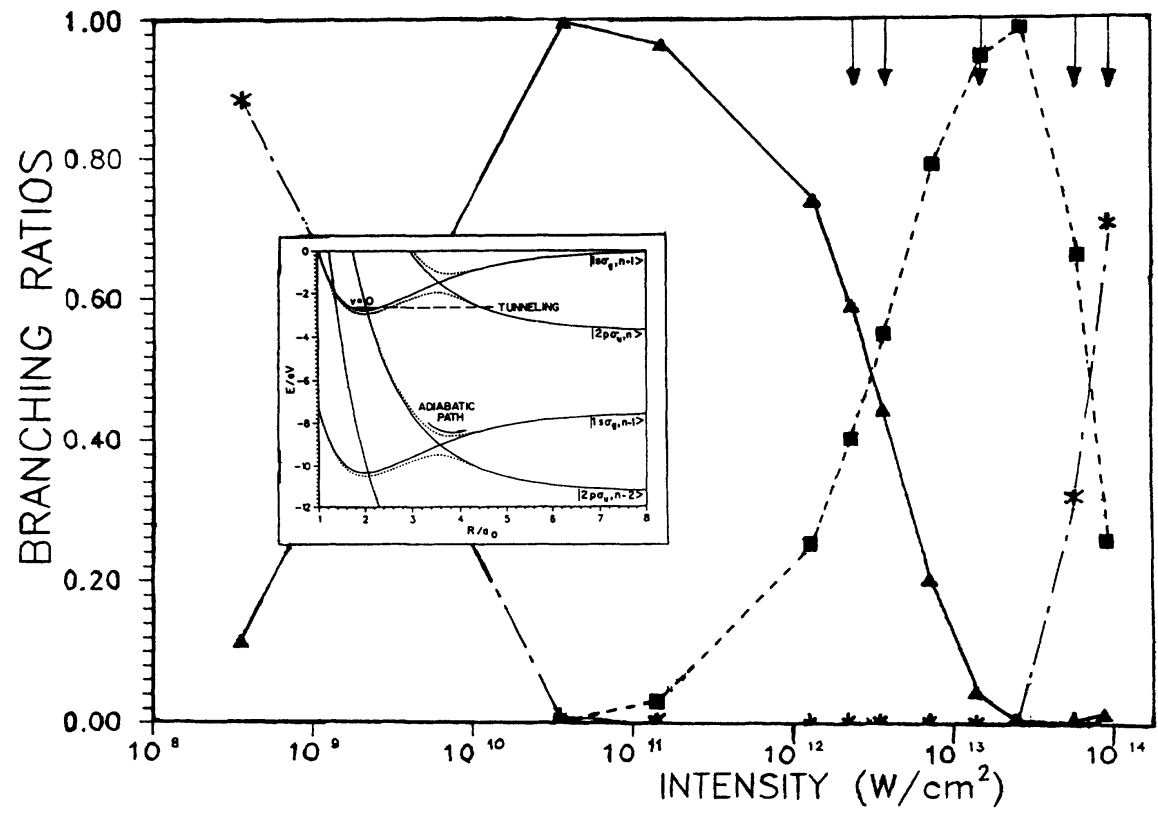

FIG. 1. Branching ratios of $\mathrm{H}_{2}{ }^{+}$as a function of the field strength over the range $10^{8}-10^{14} \mathrm{~W} / \mathrm{cm}^{2}$ for a photon wavelength $\lambda=329.7 \mathrm{~nm}$. Crosses denote the one-photon channel, squares the two-photon channel, and triangles the three-photon channel. The upper vertical arrows indicate the intensities for which the results of Table $I$ are obtained. The inset is a plot of the diabatic (full line) and adiabatic electron-plus-field potentials (dotted line) of $\mathbf{H}_{2}^{+}$with a field intensity of $1.4 \times 10^{13} \mathrm{~W} / \mathrm{cm}^{2}$ and the same wavelength. The channels bear two labels: one for the electronic degree of freedom (i.e., $1 s \sigma_{g}$ or $2 p \sigma_{u}$ ) and the other for the field (i.e., the photon number $n$ ). 
TABLE I. Branching ratios $P_{1}, P_{2}, P_{3}$ (in $\%$ ) towards channels asymptotically describing the net absorption of one, two, or three photons for five intensities $\left(I \mathrm{in} \mathrm{W} / \mathrm{cm}^{2}\right)$ and for the two isotopes $\mathrm{H}_{2}{ }^{+}$ and $\mathrm{D}_{2}{ }^{+}$, at $\lambda=329.7 \mathrm{~nm}$. $R_{32}$ (and $R_{21}$ ) are the ratios of dissociation via three-photon (or two-photon) versus the two-photon (or one-photon) channels.

\begin{tabular}{|c|c|c|c|c|c|}
\hline$I\left(\mathrm{~W} / \mathrm{cm}^{2}\right)$ & Isotope & $P_{1}(\%)$ & $P_{2}(\%)$ & $P_{3}(\%)$ & $R_{32}$ \\
\hline \multirow{2}{*}{$2.85 \times 10^{12}$} & $\mathbf{H}_{2}^{+}$ & $<10^{-1}$ & 48.17 & 51.78 & 1.07 \\
\hline & $\mathrm{D}_{2}^{+}$ & $<10^{-1}$ & 60.68 & 39.32 & 0.65 \\
\hline \multirow{2}{*}{$3.52 \times 10^{12}$} & $\mathbf{H}_{2}{ }^{+}$ & $<10^{-1}$ & 55.40 & 44.60 & 0.81 \\
\hline & $\mathrm{D}_{2}^{+}$ & $<10^{-1}$ & 68.23 & 31.74 & 0.46 \\
\hline \multirow{2}{*}{$1.40 \times 10^{13}$} & $\mathbf{H}_{2}{ }^{+}$ & $<10^{-1}$ & 95.15 & 4.84 & 0.05 \\
\hline & $\mathrm{D}_{2}^{+}$ & $<10^{-1}$ & 98.63 & 1.37 & 0.01 \\
\hline \multirow{2}{*}{$5.60 \times 10^{13}$} & $\mathrm{H}_{2}^{+}$ & 35.50 & 63.70 & 0.46 & 1.79 \\
\hline & $\mathbf{D}_{2}^{+}$ & 44.80 & 54.91 & 0.29 & 1.23 \\
\hline \multirow{2}{*}{$8.80 \times 10^{13}$} & $\mathbf{H}_{2}^{+}$ & 69.80 & 29.10 & 0.60 & 0.42 \\
\hline & $\mathrm{D}_{2}^{+}$ & 81.24 & 17.93 & 0.40 & 0.22 \\
\hline
\end{tabular}

ly, i.e., realizes in a more efficient way the diabatic jump, than the lighter $\mathrm{H}_{2}{ }^{+}$.

Let us now consider the different cases.

(i) The upper panel of Table I corresponds to the intensities of the intermediate and strong regimes where the fragmentation process is monitored by the three- and two-photon crossing channels. According to the LZ argument [Eq. (1)], the higher the radiative coupling $V_{32}$ and lower the velocity $v$, the more adiabatic the motion is. This implies that, for a given intensity, the three- to two-photon channel diabatic transition is favored for the heavier isotope. The results are in conformity with this model, i.e., $R_{32}\left(\mathrm{H}_{2}{ }^{+}\right)>R_{32}\left(\mathrm{D}_{2}{ }^{+}\right)$. The isotope effect is even enhanced with increasing intensity; the ratio $R_{32}\left(\mathrm{H}_{2}{ }^{+}\right) / R_{32}\left(\mathrm{D}_{2}{ }^{+}\right)$varies continuously from 1.65 at $I=2.85 \times 10^{12} \mathrm{~W} / \mathrm{cm}^{2}$ to 5 at $I=1.40 \times 10^{13} \mathrm{~W} / \mathrm{cm}^{2}$. Another interesting observation is that for $I=2.85 \times 10^{12}$ $\mathrm{W} / \mathrm{cm}^{2}$ the isotope substitution is at the origin of a population inversion: it is the branching ratio of the threephoton channel which dominates over the two-photon channel for $\mathrm{H}_{2}{ }^{+}$dissociation, while the reverse situation occurs for $\mathrm{D}_{2}{ }^{+}$which follows the adiabatic pathway correlating to photofragments resulting from two-photon absorption. The two other intensities $\left(3.52 \times 10^{12} \mathrm{~W} / \mathrm{cm}^{2}\right.$ and $1.40 \times 10^{13} \mathrm{~W} / \mathrm{cm}^{2}$ ) correspond, for $\mathrm{H}_{2}{ }^{+}$, to situations where $P_{2}$ is larger than $P_{3}$ (from an almost equal sharing of population for the lowest field strength, to complete population transfer in channel $\left|1 s \sigma_{g}, n-1\right\rangle$ for the highest field strength). The isotope substitution enhances these tendencies in all cases.

(ii) The lower panel of Table I gathers results from the very strong intensity regime. The fragmentation proceeds through the bond-softening mechanism leading to an important increase of $P_{1}$, the branching ratio towards the one-photon channel. The two intensities correspond to comparable populations on the two- and onephoton channels for $5.6 \times 10^{12} \mathrm{~W} / \mathrm{cm}^{2}$ and to a much more important one-photon channel population for $8.8 \times 10^{13} \mathrm{~W} / \mathrm{cm}^{2}$. The branching ratio $P_{3}$ towards the three-photon dissociation channel is negligible for the two intensities and it is the ratio $R_{21}$ of the two- to onephoton channel which is significant for the discussion. It turns out that, unexpectedly, this ratio is higher for the lighter isotope, $R_{21}\left(\mathrm{H}_{2}{ }^{+}\right)>R_{21}\left(\mathrm{D}_{2}{ }^{+}\right)$, and the isotope effect is again enhanced by the increasing intensity, i.e., $R_{21}\left(\mathrm{H}_{2}{ }^{+}\right) / R_{21}\left(\mathrm{D}_{2}{ }^{+}\right)$varies from 1.46 to 1.91 .

A simple explanation, which has repeatedly been invoked when very strong fields are assumed, is a barrier lowering of the adiabatic electronic potential diagonalizing the radiative coupling $[3,4,6]$. Such an effect monitors the decay of the shape resonance $(v=0, j=1)$ by tunneling through the adiabatic channel correlating to fragments with kinetic energy corresponding to a onephoton absorption $\left|2 p \sigma_{u}, n\right\rangle$, the whole process being dominated by a single-photon exchange between the molecule and the electromagnetic field. With respect to isotope substitution, the decay by tunneling is favored for the lighter $\mathrm{H}_{2}{ }^{+}$(smaller mass factor) which, in addition, experiences a lower barrier (higher vibrational frequency). Such a model would lead to $R_{21}\left(\mathrm{H}_{2}{ }^{+}\right)<R_{21}\left(\mathrm{D}_{2}{ }^{+}\right)$, the inequality being enforced with increasing intensity resulting into barriers which flatten accordingly. This is clearly in contradiction with our findings. Conversely, the results displayed in Table I can, at least partially, be interpreted in terms of a multiphoton process involving three steps. An initial three-photon ab- 
sorption can be modeled referring to a $\mathrm{LZ}$ type of argument with an "effective" coupling between diabatic channels $\left|1 s \sigma_{g}, n+1\right\rangle$ and $\left|2 p \sigma_{u}, n-2\right\rangle$, indirectly coupled at the curve-crossing position $R \simeq 2$ a.u. For this first step the transition probability is larger for the heavier isotope $\mathrm{D}_{2}{ }^{+}$. The second step corresponds to a photon emission which occurs at $R=3.6$ a.u., the position of the second curve crossing between radiatively coupled diabatic channels $\left|2 p \sigma_{u}, n-2\right\rangle$ and $\left|1 s \sigma_{g}, n-1\right\rangle$. Here again the LZ argument favors the transition for the heavier isotope. The last step is a subsequent photon emission leading to a transition from the diabatic channel $\left|1 s \sigma_{g}, n-1\right\rangle$ to $\left|2 p \sigma_{u}, n\right\rangle$ without any curve crossing. The LZ formula of Eq. (1) cannot be transposed directly to such a case, but one can presumably argue that the heavier isotope is again favored for this transition, namely because its lower velocity is such that actually $\mathrm{D}_{2}{ }^{+}$is exposed to the radiative field for a longer time than $\mathrm{H}_{2}{ }^{+}$. In summary, all three steps contribute to the enhancement of the diabatic transition probability of $\mathrm{D}_{2}{ }^{+}$with respect to $\mathrm{H}_{2}{ }^{+}$, in complete agreement with the results of
Table I. This shows that the above-described five-photon mechanism is more likely than the single-photon tunneling as has already been mentioned [10,11].

As a word of conclusion, we consider the effect of isotope substitution in the $\mathrm{H}_{2}{ }^{+}-\mathrm{D}_{2}^{+}$photodissociating system as an additional proof to the fact that the one-photon dissociation process cannot merely be interpreted in terms of bond softening by a field-dressed barrier lowering, described by a single-photon exchange mechanism. We rather suggest that this be interpreted in terms of a competition between the single-photon mechanism and a five-photon mechanism involving three simultaneously absorbed and two successively emitted photons.

This work was funded by an International SpainFrance Collaboration Project. One of us (O.A.) also acknowledges partial support from France-Québec ESR Project No. 010392. The Laboratoire de Photophysique Moléculaire is "associé au Centre National de la Recherche Scientifique."
[1] Coherent Phenomena in Atoms and Molecules in Laser Fields, Vol. 287 of NATO Advanced Study Institute, Series B: Physics, edited by A. D. Bandrauk and S. C. Wallace (Plenum, New York, 1992).

[2] A. Giusti-Suzor, X. He, O. Atabek, and F. H. Mies, Phys. Rev. Lett. 64, 515 (1990).

[3] A. D. Bandrauk, E. Constant, and J. M. Gauthier, J. Phys. II France 1, 1033 (1991).

[4] G. Jolicard and O. Atabek, Phys. Rev. A 46, 5845 (1992).

[5] A. D. Bandrauk, E. Aubanel, and J. M. Gauthier, Laser Phys. 3, 381 (1993).

[6] P. H. Bucksbaum, A. Zavriyev, H. G. Muller, and D. W. Schumacher, Phys. Rev. Lett. 64, 1883 (1990).

[7] A. Giusti-Suzor and F. H. Mies, Phys. Rev. Lett. 68, 3869 (1992).

[8] O. Atabek, M. Chrysos, and R. Lefebvre, Phys. Rev. A 49, R8 (1994).

[9] B. Yang, M. Saeed, L. F. Dimauro, A. Zavriyev, and P. H. Bucksbaum, Phys. Rev. A 44, R 1458 (1991).
[10] M. Chrysos, O. Atabek, and R. Lefebvre, Phys. Rev. A 48, 3845 (1993); 48, 3855 (1993).

[11] O. Atabek and G. Jolicard, this issue, Phys. Rev. A 49, 1186 (1994).

[12] S. Chelkowski, T. Zuo, and A. D. Bandrauk, Phys. Rev. A 46, R5342 (1992).

[13] F. V. Bunkin and I. I. Tugov, Phys. Rev. A 8, 601 (1973).

[14] S. Miret-Artés, O. Atabek, and A. D. Bandrauk, Phys. Rev. A 45, 8056 (1992).

[15] A. D. Bandrauk and G. Turcotte, J. Chem. Phys. 89, 3039 (1985); A. D. Bandrauk and O. Atabek, in Lasers, Molecules and Methods, edited by J. O. Hirschfelder, R. E. Wyatt, and R. D. Coalson (Wiley, New York, 1989), Chap. 19.

[16] M. Shapiro, J. Chem. Phys. 56, 2582 (1972).

[17] L. D. Landau and E. M. Lifshitz, Quantum Mechanics (Pergamon, New York, 1965); M. S. Child, Semiclassical Mechanics with Molecular Applications (Oxford University Press, New York, 1991), pp. 62-63. 